\title{
Responsabilidad social empresarial \\ en la jurisprudencia de la Corte \\ Constitucional colombiana: dimensiones \\ de obligatoriedad en la voluntariedad
}

\author{
Corporate Social Responsibility in the Jurisprudence of the Colombian \\ Constitutional Court: Binding Dimensions within Voluntariness
}

Responsabilidade social empresarial na jurisprudência do Tribunal

Constitucional colombiano: dimensões do voluntário obrigatório

\author{
LINA LORENZONI ESCOBAR*
}

FECHA DE RECEPCIÓN: 8 DE ABRIL DE 2020. FECHA DE APROBACIÓN: 5 DE OCTUBRe De 2020

Doi: https://doi.org/10.12804/revistas.urosario.edu.co/sociojuridicos/a.9085

Para citar este artículo: Lorenzoni Escobar, L. Responsabilidad social empresarial en la jurisprudencia de la Corte Constitucional colombiana: dimensiones de obligatoriedad en la voluntariedad. Revista de Estudios Socio-Jurídicos, 23(1), 347-369. https://doi.org/10.12804/revistas.urosario.edu.co/sociojuridicos/a.9085

\section{RESUMEN}

El propósito de este artículo es determinar si la normatividad y esquemas que materializan la responsabilidad social empresarial (RSE) se traducen en obligaciones para las empresas activas en el territorio colombiano. Para el desarrollo de este objetivo, se llevó a cabo un estudio general del concepto de RSE: su origen, desarrollo y estructura, con apoyo en la doctrina y en algunos instrumentos de RSE internacionales. En particular, se evidencia cómo el debate internacional sobre esta oscila entre intentos por volverla obligatoria e instrumentos donde se manifiesta como una herramienta normativa blanda. Este fenómeno es evidente también en el plano doméstico colombiano, donde la jurisprudencia constitucional se ha ocupado de la RSE y de su relación con el ordenamiento constitucional colombiano. A través de esta jurisprudencia, se identifica el respaldo constitucional de la RSE en Colombia, así como el entendimiento

* Docente de derecho económico internacional en la Universidad de Antioquia, Medellín. Licenciada en derecho de la Universidad de Trento (Italia), abogada en Colombia. Magíster en derecho internacional de la Universidad de Heidelberg (Alemania). Especialista en derecho administrativo de la Universidad de Antioquia. Candidata a doctor en derecho internacional de la Universidad Martín Lutero de Halle Wittenberg (Alemania). Correo electrónico: lina. lorenzoni@udea.edu.co ORCID: https://orcid.org/0000-0003-3632-6603 
sobre su alcance. Se concluye que, si bien el juez constitucional reitera que la RSE es voluntaria, la narrativa constitucional de esta contiene elementos de obligatoriedad.

Palabras clave: responsabilidad social empresarial; Corte Constitucional; sostenibilidad; obligatoriedad; voluntariedad.

\section{ABSTRACT}

The purpose of the present article is to determine whether the normativity and schemes of corporate social responsibility (CSR) translate into binding obligations for companies operating in Colombia. To this end, this article carries out a general study of the concept of CSR: its origin, development, and structure, based on the doctrine and certain international CSR instruments. In particular, this article highlights how the international debate on CSR fluctuates between attempts to make it binding and instruments where it is developed as soft law. This phenomenon is also evident in Colombian domestic law, where the jurisprudence of the Constitutional Court has addressed CSR and its relationship with the Colombian constitutional order. This jurisprudence identifies the constitutional backbone for CSR, as well as the constitutional understanding of its reach. This article concludes that, while the Constitutional Court reiterates that CSR is voluntary, the constitutional narrative on CSR contains binding elements.

Keywords: Corporate Social Responsibility; Constitutional Court; Sustainability; Binding; Voluntary.

\section{RESUMO}

O objetivo deste artigo é determinar se os regulamentos e esquemas que materializam a responsabilidade social empresarial (RSE) se traduzem em obrigações para as empresas que atuam no território colombiano. Para o desenvolvimento deste objetivo, foi realizado um estudo geral do conceito de RSE, especificamente, a sua origem, desenvolvimento e estrutura, com o apoio da doutrina e de alguns instrumentos internacionais de RSE. Particularmente, se evidencia como o debate internacional sobre a RSE oscila entre as tentativas de torná-la obrigatória e os instrumentos onde ela se manifesta como uma ferramenta normativa fraca. Este fenômeno também é evidente na esfera interna colombiana, onde a jurisprudência constitucional tem tratado da RSE e de sua relação com a ordem constitucional colombiana. Por meio desta jurisprudência, identifica-se o respaldo constitucional da RSE na Colômbia, bem como a compreensão de seu alcance. Conclui-se que, embora o juiz constitucional reitere que a RSE é voluntária, a narrativa constitucional da RSE contém elementos de obrigatoriedade.

Palavras-chave: responsabilidade social empresarial; tribunal constitucional; sustentabilidade; obrigatoriedade; voluntariedade. 


\section{Introducción}

El rol de las empresas en nuestras sociedades ha evolucionado a la par con el del Estado en el contexto globalizado. Lejos está el Estado de tener la capacidad de garantizar la totalidad de derechos en cabeza de sus ciudadanos, mientras que la empresa, como base del desarrollo, ha evidenciado su importante papel en la generación de bienestar (Corte Constitucional, T-375), a través de la distribución de bienes y servicios que inciden sobre el ambiente y los derechos humanos de sus trabajadores y de las comunidades vecinas a sus actividades (Correa $\&$ Roa, 2019). Esto es especialmente evidente en países como Colombia, en aquellos rubros de la economía, como la minería y la extracción de hidrocarburos, que se desarrollan en áreas rurales, en las cuales históricamente la presencia del Estado ha sido deficiente. Críticas al actuar de las empresas en estos contextos han dado pie, a nivel global, a un movimiento que busca regular el comportamiento empresarial y definir ciertos estándares plenamente exigibles a las empresas.

El auge que ha tenido esta aspiración en el plano internacional ha tenido repercusiones en el plano interno porque las empresas que operan en el territorio adoptan esquemas y políticas de RSE, y porque esta se integra al discurso del legislador y de la jurisprudencia colombiana sobre el tema. Se ha generado así un diálogo entre fuentes del derecho ortodoxas, como la legislación y la jurisprudencia, y manifestaciones regulatorias blandas - soft law-, término que suele usarse para describir la naturaleza de aquellas iniciativas en las que intervienen con calidad de reguladores actores no estatales como las empresas (Chinkin, 1980) o de aquellas iniciativas en las que intervienen Estados, que no buscan generar obligaciones vinculantes (Klabbers, 1996).

En particular, es la jurisprudencia de la Corte Constitucional, preponderantemente a través de acciones de tutela, la que ofrece puntos de análisis para ver cómo dicha corporación se ha apropiado de la narrativa internacional de la RSE y qué implicaciones tiene esto en las operaciones de las empresas en Colombia, tanto de cara a la existencia de contenidos mínimos de RSE, como a su posible exigibilidad. Así, el entendimiento de la Corte Constitucional acerca de la RSE se complementa con la narrativa que sobre la misma existe en el plano internacional, generando 
ciertas dimensiones de obligatoriedad en virtud de su conexión con los principios constitucionales que rigen el sistema jurídico colombiano. Estas dimensiones se articulan preponderantemente a partir de iniciativas internacionales de soft law, poniendo de manifiesto una fertilización cruzada (Linderfalk, 2014) en la que intervienen un acervo regulatorio internacional no ortodoxo y el ordenamiento jurídico colombiano. Así, el "endurecimiento" de dichas fuentes blandas podría estarse dando no en el plano internacional, sino en el interno colombiano a través de la jurisprudencia de la Corte Constitucional.

Adicionalmente, al evidenciar la aparente paradoja de dimensiones de obligatoriedad en el marco de la voluntariedad, la Corte Constitucional obliga a matizar, para el contexto colombiano, la idea que la RSE sea un ejercicio arbitrario de las empresas. En efecto, estas dimensiones de obligatoriedad contribuyen a una narrativa nacional de la RSE en la que la aplicación de estándares internacionales se aparta de las percepciones subjetivas de los grupos de interés, para generar cierta predictibilidad en torno a qué debe cumplir una empresa en su política de RSE (Aftab E Mocle, 2019).

El presente artículo se propone analizar dicha narrativa e identificar dichas dimensiones. Para esto, inicialmente se presentará un análisis de la RSE en el plano internacional, a partir de una revisión doctrinaria y del actual estado de las iniciativas que regulan o buscan regular el actuar de la empresa. En particular, se destaca el tipo de proceso de regulación que genera la RSE a nivel internacional, y cómo este se diferencia de la obligatoriedad que caracteriza las fuentes formales del derecho. Este análisis evidencia cómo el debate internacional en torno a la RSE se caracteriza por oscilar entre intentos por volverla obligatoria e instrumentos donde se manifiesta como una herramienta normativa blanda, donde estos últimos son sin duda los que predominan y tienen mayor acogida.

En un segundo momento, a través del análisis de la jurisprudencia de la Corte Constitucional, se identifican los fundamentos constitucionales para la RSE en Colombia, su alcance y la forma como debe entenderse la voluntariedad de la RSE. En este sentido, se hace un análisis jurisprudencial a partir del cual se reconstruye la RSE en el marco constitucional colombiano, a la vez que se evidencia la arriba mencionada fertilización 
cruzada entre fuentes blandas y tradicionales del derecho, enmarcadas bajo la misma narrativa jurisprudencial. Finalmente, se concluye que, aunque la Corte Constitucional es enfática en destacar la dimensión voluntaria y autorreguladora de la RSE, a través de la conexión de esta última con el ordenamiento constitucional colombiano, existen límites y cierta obligatoriedad de contenidos que se escapan de un entendimiento voluntario en sentido absoluto.

\section{La RSE y su origen internacional: entre voluntariedad y obligatoriedad}

Referencias a la RSE aparecen en la literatura desde 1930 (Carroll, 1999). Desde 1950 se encuentran las primeras discusiones sobre el alcance específico de las responsabilidades de las empresas hacia la sociedad (Latapí Agudelo et al., 2019). Sin embargo, los procesos regulatorios, especialmente internacionales, asociados a la RSE, tienen su auge con la globalización de la economía (Okoye, 2016), pues son el resultado de la liberalización del comercio y de las inversiones ( $\mathrm{Mu}-$ chlinski, 2011), a la vez que reflejan una creciente preocupación del público por el impacto social y ambiental del crecimiento económico (Latapí Agudelo et al., 2019).

La teorización de la RSE ha evolucionado notablemente desde la famosa concepción de Milton Friedman (1970), eminentemente centrada en utilidades para los accionistas, quien la teorizó como "el uso de sus recursos [los de la empresa] y el manejo de sus actividades para el aumento de sus utilidades, siempre y cuando respete las reglas del juego" (p. 3)1. Desde entonces, la consciencia de que el funcionamiento de una empresa no solo afecta positiva y negativamente los intereses de sus accionistas, sino también los de otros grupos, como trabajadores, clientes, consumidores y la comunidad donde está asentada (BonillaSanabria, 2017) ha reforzado la necesidad de teorizar acerca de una responsabilidad empresarial por las consecuencias de dichas afectaciones

1 Traducción propia. 
negativas, lo que por su parte ha dado pie a sendas iniciativas de regulación en sede internacional.

Estas iniciativas, en el plano internacional, son de dos tipos. Por un lado, encontramos aquellas que buscan regular la responsabilidad internacional de las empresas a través de la creación de obligaciones internacionales para las mismas, idealmente consagradas en tratados internacionales. Se trata entonces de iniciativas que buscan generar una responsabilidad internacional a partir de fuentes tradicionales del derecho internacional. El más reciente esfuerzo en este sentido está actualmente en curso, auspiciado por el Grupo de Trabajo Intergubernamental de Composición abierta sobre las Empresas Transnacionales y Otras Empresas Comerciales en Materia de Derechos Humanos (Naciones Unidas, 2014), que busca un tratado internacional sobre empresas y derechos humanos. Dicho proyecto, que ya cuenta con un segundo borrador (López, 2019), tiene su predecesor en las Normas sobre las Responsabilidades de las Empresas Transnacionales y Otras Empresas Comerciales en la Esfera de los Derechos Humanos (Naciones Unidas, 2003), en el sentido de que dichas normas pretendían teorizar, aunque no en el marco de un tratado, obligaciones para empresas transnacionales, lo que generó un debate que las hizo fracasar (Rodríguez, 2017).

Por otro lado, encontramos aquellas iniciativas, como el Pacto Global (Naciones Unidas, 2000) y los Principios Rectores (Naciones Unidas, 2011), que se caracterizan por ser el resultado de procesos en los que intervienen Estados, pero cuyo resultado no es un tratado internacional. Por tanto, dichas iniciativas, al ser de soft law, se apartan de las consecuencias asociadas a tratados ratificados y en vigor; en particular, se apartan del atributo de obligatoriedad.

De manera similar, actores no estatales, como organizaciones no gubernamentales, han generado iniciativas igualmente influyentes como las Líneas Directrices para Empresas Multinacionales de la Organización para el Desarrollo y la Cooperación Económica (OCDE), cuya primera versión es de 1976 y la más reciente de 2011. En este sentido, se manifiesta el soft law ya no desde los Estados, sino desde los actores no estatales que, en principio, no generarían fuentes vinculantes de derecho.

Existen una multitud de iniciativas internacionales, resultado de los compromisos gestados por empresas, Estados, otros grupos de interés 
o cualquier nivel de intersección entre estos últimos, consagradas en declaraciones como las ya expuestas, en sellos o en validadores de conducta, como el ISO 26000, o en herramientas para reportar la sostenibilidad, como la Global Reporting Initiative (GRI).

Este segundo tipo de iniciativas de soft law tienen diversos niveles de incidencia. El Pacto Global y los Principios Rectores se caracterizan por su amplia acogida por parte de las empresas y sus gremios. Es decir, los esquemas de regulación que se caracterizan por la no obligatoriedad - soft law - son aquellos que han incidido en la práctica de la RSE. También son los esquemas a los cuales se ha referido la Corte Constitucional en sus teorizaciones sobre la naturaleza de la RSE como voluntaria, en particular, el Pacto Global (Corte Constitucional, T-247) y los Principios Rectores de Naciones Unidas (Corte Constitucional, T-372).

Como ya se ha mencionado, el origen de dichos esquemas no refleja modos de creación del derecho tradicional, más bien son la manifestación de una "gobernanza experimentalista" (Rodríguez, 2017), es decir, una gobernanza policéntrica (Fischer-Lescano \& Teubner, 2004) que, a diferencia de la estatal, se caracteriza por el concierto de una diversidad de actores, entre ellos Estados, sociedad civil y empresas, para regular áreas específicas del conocimiento (Fischer-Lescano \& Teubner, 2004). En este sentido, las empresas han mostrado que, junto con ser destinatarias de normas, también son arquitectas de estas (Correa \& Roa, 2019). Así, este tipo de gobernanza evidencia que en ausencia de un rol regulador absoluto del Estado, surge una regulación fragmentada (Teubner, 2017), funcional al conocimiento, que se manifiesta mediante modos de producción y resultados distintos a los tradicionales (Picciotto, 2002).

El panorama brevemente esbozado muestra que la disciplina de la RSE tiene su origen en el plano internacional y que es a partir de ahí que entra a ser objeto de discusión en el foro doméstico de los países. Es por esto que esta discusión se ha enmarcado, también a nivel de la jurisdicción interna colombiana, en términos de voluntariedad y obligatoriedad, debido a la postura dominante, aunque en evolución, sobre la falta de subjetividad internacional de las empresas y la imposibilidad de adscribirles derechos y obligaciones de tipo internacional (Tomuschat, 2014). Dicha imposibilidad dogmática explica también, en parte, el fracaso de las iniciativas de regulación de la responsabilidad 
empresarial bajo forma de obligaciones consagradas en tratados internacionales (Ruggie, 2017).

El hecho que la RSE no se traduzca en obligaciones exigibles en el sentido tradicional también depende del constante estado de evolución de las circunstancias a las cuales debe responder la RSE, lo que hace difícil acomodarla de acuerdo con el esquema de normatividad vinculante (Okoye, 2016). Lo anterior ha sido objeto de críticas puesto que se identifica la falta de carácter obligatorio o vinculante de la RSE, con una adherencia puramente voluntaria y una violación exenta de consecuencias para las empresas (Alfonso, 2013; Tamvada, 2019). Así, al no ser vinculantes para las empresas dichas iniciativas, la crítica encuentra que "diluyen su fuerza ante el destinatario de la norma" (Acevedo et al., 2013).

Sin embargo, hay que matizar los términos "voluntario" y "falto de consecuencias" en el contexto de la RSE. En efecto, en el plano internacional, a la vez que se discute una norma convencional y vinculante para las empresas en el seno del Grupo de Trabajo Intergubernamental de Composición abierta sobre las Empresas Transnacionales y Otras Empresas Comerciales en Materia de Derechos Humanos, también empiezan a surgir, en particular en el arbitraje de las inversiones, reflexiones en torno a una subjetividad internacional para las empresas y, por tanto, una obligatoriedad de sus iniciativas de RSE (Urbaser c. Argentina, 2016). Por otro lado, en las jurisdicciones domésticas de los países, se dan debates sobre las implicaciones de las iniciativas de RSE "voluntarias" en el derecho interno.

En el caso colombiano, es la jurisprudencia de la Corte Constitucional la que ofrece claves de lectura para entender la RSE aplicada al contexto del país, a la vez que permite evidenciar el diálogo entre la Corte y los otros poderes del Estado (Correa \& Roa, 2019); en especial, entre la Corte y las instancias internacionales que han desarrollado la RSE - la fertilización cruzada arriba mencionada-. Por un lado, de dicha jurisprudencia se desprende un fundamento constitucional para la RSE, junto con sus alcances y fines constitucionales. La Corte encuentra dicho fundamento en la Constitución, pero lo elabora teóricamente a partir del marco del soft law internacional sobre la materia. Por otro lado, en la narrativa de la Corte Constitucional sobre RSE se evidencia 
cómo dicha corporación maneja los conceptos de voluntarismo y de obligatoriedad, generando un espacio entre ambos del que se desprenden pautas de comportamiento que no son enteramente voluntarias para las empresas. Así, junto con el dualismo entre lo voluntario y lo obligatorio, existe una categoría que no se identifica ni con lo primero ni con lo segundo, una paradoja conceptual que indica también una nueva forma de concebir procesos cognitivos (Neuwirth, 2017) y de interpretar realidades.

\section{Jurisprudencia constitucional y RSE: fundamentos constitucionales, alcances y fines}

La Corte Constitucional ha identificado dos fundamentos constitucionales para la RSE en el ordenamiento colombiano: la función social de la actividad empresarial y el principio de solidaridad. La función social de la empresa, como base del desarrollo, que implica obligaciones, está consagrada explícitamente en la Constitución, la cual también establece que la libre competencia es un derecho que supone responsabilidades (Constitución Política, artículo 333). De esta manera, la función social implica que las empresas, además de producir riqueza, tienen obligaciones que son consustanciales, de naturaleza social (Acevedo et al., 2013) - una manifestación del modelo económico adoptado por el Constituyente en 1991- y de economía social de mercado (MorgensteinSánchez, 2019).

Efectivamente, la Corte Constitucional ha indicado que justamente debido a que las empresas satisfacen importantes necesidades de la comunidad, la libertad económica que la Constitución les asigna coexiste con una función social que implica obligaciones (Corte Constitucional, T-375). De esta manera, la legitimidad de las decisiones que tome una empresa debe analizarse a través de esta "doble dimensión", que obliga a mirar el prisma de la autonomía a partir de dichas decisiones, a la luz de las consecuencias sociales y ecológicas de las mismas (Corte Constitucional, T-375). En este sentido, la Carta Política (artículo 333) concilia la aspiración de enriquecimiento de los empresarios con los 
derechos de otros grupos de personas que pueden verse afectados por la actividad económica (Morgenstein-Sánchez, 2019).

Por tal razón, al estudiar la constitucionalidad de las cláusulas de RSE incluidas en algunos tratados de libre comercio (TLC) la Corte las ha declarado exequibles, en la medida en que alentar las "prácticas voluntarias de responsabilidad social corporativa" es parte de la función social de la empresa, consagrada en el artículo 333 de la Constitución (Corte Constitucional, C-915). Este fundamento constitucional de la RSE fue reiterado por la corporación cuando afirmó que la RSE, si bien no se encuentra explícitamente establecida en la Constitución, encuentra su base constitucional "sólida, suficiente y autónoma" en el artículo 333 de la Carta (Corte Constitucional, T-781, párrafo 4.5.3.). Este fundamento constitucional es el que, en palabras de la Corte, contribuye a que la RSE sea necesaria y, a la vez, un compromiso voluntariamente adquirido (Corte Constitucional, T-247), una paradoja que se analizará en la tercera parte de este escrito.

Por otro lado, la solidaridad se encuentra enunciada en varios artículos de la Constitución. En particular, obrar conforme al principio de solidaridad social es un deber de toda persona y ciudadano (Constitución Política, artículo 95). En este sentido, la solidaridad ha sido articulada por la Corte Constitucional como principio y deber. Como deber obliga a los particulares a vincular el esfuerzo propio en apoyo de otros (Quinche, 2009) o del interés colectivo (Corte Constitucional, 2014, C-767). El principio de solidaridad, por tanto, ha incidido sobre el principio de autonomía privada, limitándola en particular en sectores que desarrollan actividades de interés público (Bernal, 2016).

La Corte Constitucional ha identificado una "íntima conexión" entre la RSE y el principio de solidaridad, en la medida que ambos concretan un deber constitucional que le permite a los actores influir en el desarrollo de derechos fundamentales (Corte Constitucional, T-247). Igualmente, la Corte ha afirmado, también en sede de estudio de constitucionalidad de cláusulas de RSE en un TLC, que incluir los principios de RSE en el tratado coadyuva al cumplimiento de principios constitucionales como la solidaridad (Corte Constitucional, C-608). De esta manera, el principio constitucional de solidaridad y la función social de la empresa se manifiestan concretamente en la RSE. Aquí encontramos entonces un primer 
nivel de coexistencia entre un precepto que es obligatorio, bien sea el principio de solidaridad o la función social de la actividad empresarial, directamente consagrados en la Constitución, con una manifestación de dichos preceptos que sería voluntaria y autorregulada, es decir, la RSE.

No obstante, la forma como se manifiestan los fundamentos constitucionales en la RSE no sería enteramente voluntaria, en la medida en que la Corte también se ha pronunciado sobre el contenido de la RSE. Cabe anotar que se trata de un contenido que obedece a las afectaciones que causan actividades empresariales lícitas y necesarias (Corte Constitucional, T-732), en el entendido de que los particulares, entre ellos las empresas, pueden afectar derechos humanos y el medio ambiente. La Corte parece entonces construir su aproximación a la RSE a partir de la "relación de impacto" que se puede generar entre un actuar empresarial y el entorno en el que opera (Tamvada, 2020).

A partir de esta relación de impacto, la Corte ha indicado cuáles son los contenidos, sustantivos y procesales, de la RSE. El énfasis sobre el contenido de la RSE debe leerse en el marco de la preocupación generalizada, a nivel global, sobre el llamado "lavado verde" (greenwashing en inglés), por parte de empresas que, de manera deliberada, adoptan el lenguaje de la sostenibilidad sin reflejar sus afirmaciones, ambientales y sociales, en su práctica o incluso, para distraer de su real desempeño ambiental o social (Gatti et al., 2019). Lo anterior ocurre por una ausencia de estandarización de la RSE (Gatti et al., 2019), concebida como un ejercicio netamente voluntario sin mecanismos de verificación ni exigibilidad (Tamvada, 2020). Por esto mismo, se ha sostenido que el lavado verde podría prevenirse mediante una refinación del sistema regulatorio de la RSE, ya sea a través del desarrollo de estándares o la integración de las dimensiones de lo voluntario y lo obligatorio (Gatti et al., 2019). En este sentido, la Corte se alinea con esta última tendencia, en la medida en que busca refinar la regulación de la RSE introduciendo un marco de referencia, el cual, sin embargo, no niega la dimensión voluntaria. De esta manera, la Corte distingue un marco sustantivo de un marco procesal para la RSE.

Los elementos sustantivos de la RSE se materializan en la protección del medio ambiente y en la mejora social o desarrollo humano integral, en el marco de las operaciones de una empresa: pilares ambiental y 
social (Corte Constitucional, T-247; T-781; T-061). Lo anterior incluye el respeto por parte de la empresa de los derechos humanos. Efectivamente, la Corte indica que en un Estado social de derecho, "los derechos no generan únicamente obligaciones en cabeza del Estado" y que las empresas "deben actuar de conformidad con la protección al ambiente y los derechos de las personas que habitan en las zonas en las que pueden generar alguna afectación" (Corte Constitucional, T-732, párrafo 52). Los pilares ambientales y sociales de la RSE podrán ser administrados por las empresas mediante esquemas como el Global Compact (Corte Constitucional, T-247), los cuales no pierden su característica de voluntariedad; en este sentido, debe interpretarse que la empresa podrá optar por un esquema de gestión de sus impactos ambientales y sociales sin ser obligatorio uno en particular.

Los elementos que podríamos definir como procesales, en relación con los grupos de interés, consisten en la "interacción con los agentes interesados" (Corte Constitucional, T-781). La Corte reconoce entonces que la empresa tiene responsabilidades con los grupos de interés. La Corte no ofrece ningún criterio para la identificación de los grupos de interés y sobre este punto la literatura tiende a considerar como grupos de interés, aquellos grupos e individuos que pueden afectar a la empresa o ser afectados por ella (Freeman et al., 2010). Así, la teoría de los grupos de interés implica integrarlos al modelo de negocios (Freeman et al., 2010). Aún así, hay varias aproximaciones a la identificación de los grupos de interés (Mitchell \& Lee, 2019), sin que la Corte Constitucional haya sido específica sobre ninguna en particular, de manera que, en este ejercicio de identificación de los mismos, la Corte se remite a la discreción de las empresas. La remisión es entonces similar a la ya vista para el eje sustancial, en la medida en que la obligatoriedad del eje se traduce en opciones de gestión para las empresas.

Lo anterior debe, sin embargo, matizarse en el contexto mineroenergético, donde la Corte es un poco más específica en la descripción de la interacción con los grupos de interés, al indicar que se trata de incluir en las políticas de RSE mecanismos de participación con las comunidades y relacionamiento con las autoridades locales (Corte Constitucional, Su-095). También en el contexto minero-energético, la Corte habla de "empresas como" Ecopetrol, donde el elemento procesal se manifiesta 
adicionalmente en los procesos internos de la empresa en relación con su RSE, que se deben regir por el principio de transparencia (Corte Constitucional, T-061), de manera que no haya prácticas encubiertas o vulneraciones indirectas (Corte Constitucional, T-247).

Estos dos elementos - el substantivo y el procesal-deberán en todo caso guardar conformidad con el espíritu de la Constitución Política (Corte Constitucional, T-781) y los objetivos del Estado social (Corte Constitucional, T-247). El fin último de la RSE consistirá en que la empresa pueda promover el bienestar social de la comunidad (Corte Constitucional, T-247), mediante la realización del contenido de los derechos fundamentales de las personas y comunidades que se encuentran en las áreas de impacto donde ejecutan sus actividades (Corte Constitucional, T-781). La Corte reconoce que la empresa es un "sujeto que actúa al interior de una sociedad" (Corte Constitucional, T-247, parágrafo 7), de la cual deriva una riqueza económica (Corte Constitucional, T-129), que por tanto debe ser partícipe de su desarrollo y promotor de su bienestar (Corte Constitucional, T-247; T-129).

En este sentido, resalta la Corte que los programas de RSE cumplen un rol distinto pero complementario al del Estado, puesto que, desde una perspectiva distinta a la de este, persiguen sus mismos objetivos: operan sobre el mismo telos (Corte Constitucional, T-247). En la RSE se identifica una concurrencia (Correa y Roa, 2019), compatibilidad (Corte Constitucional, T-247), complementariedad (Corte Constitucional, T-781) y armonía (Corte Constitucional, T-061) con los fines y la configuración del Estado social de derecho.

\section{Jurisprudencia constitucional y la naturaleza de la RSE: ¿voluntaria u obligatoria?}

La Corte Constitucional se refiere a la RSE como una forma de "autorregulación", una serie de políticas o lineamientos que las empresas "suelen adoptar de forma discrecional o facultativa, en forma de soft law" (Corte Constitucional, T-781, párrafo 4.5.1).

Este entendimiento de la RSE como un ejercicio discrecional o facultativo es también como la Corte ha analizado herramientas específicas, 
como los principios del Pacto Global o los Principios Rectores. En efecto, la Corte ha sido enfática en resaltar que los principios del Pacto Global son voluntarios, entendidos como "no vinculantes"; es decir, dependen de sus destinatarios, sus compromisos y su autoevaluación para su aplicación (Corte Constitucional, T-247). En relación con los Principios Rectores, la Corte también ha indicado, parafraseando la advertencia contenida en los mismos, que no son un "nuevo tratado de derechos humanos, no crean derechos o nuevas obligaciones" (Corte Constitucional, T-732, párrafo 52). Así, se desprende de estas premisas lo que la RSE y sus instrumentos no son; no son vinculantes. Es más, la RSE es algo que "trasciende el simple cumplimiento de las normas" (Corte Constitucional, T-781, párrafo 4.5.1.), va "más allá del mero cumplimiento de la obligación legal" (Corte Constitucional, T-129, párrafo 9.7.6.): a contrario, no se trataría de una obligación legal. Sin embargo, esta voluntariedad $a b$ origine de los principios no se traduce, necesariamente, en una voluntariedad en la forma como deben ser aplicados en Colombia.

Efectivamente, la Corporación ha destacado que los principios de RSE, al tener elementos incluidos en la regulación constitucional, "resultan vinculantes por cuanto normas de naturaleza constitucional" (Corte Constitucional, T-247, párrafo 7), y que no se puede desconocer la relación estrecha de los compromisos "voluntarios" de RSE con los postulados y fines constitucionales (Corte Constitucional, T-781). En este sentido, la voluntariedad de la fuente de la RSE, en la práctica, está enmarcada y obligatoriamente delimitada en virtud de su conexidad con los principios constitucionales. De esta manera, las fuentes de soft law invocadas por la Corte para sustentar una voluntariedad ab origine de la RSE, en realidad se "endurecen" al ingresar el ordenamiento constitucional colombiano, en aquellos puntos donde se superponen a los postulados y fines de la Carta.

La Corte insiste en que el hecho que las empresas operen en el marco de un Estado social de derecho las obliga a actuar de conformidad con la protección del medio ambiente y los derechos de las comunidades de las zonas donde operan (Corte Constitucional, T-732), pues no podría tolerarse la vulneración por parte de particulares de derechos de cuya realización dependen los fines del Estado. En este sentido, la Corte ha identificado los dos ejes sustanciales: el ambiental y el social, y un eje 
procesal, orientado al diálogo y a la transparencia con las comunidades, de la RSE. Estos ejes deben interpretarse por parte de las empresas como un de minimis para una política de RSE que cumpla con los lineamientos constitucionales del ordenamiento colombiano. Este de minimis, en el caso de las empresas del sector minero-energético, está reforzado no solo en la exigencia de transparencia con comunidades y autoridades locales, sino también en el riguroso seguimiento que sobre las políticas de RSE adoptadas deberán adelantar las autoridades minero-ambientales.

De esta manera, la RSE, así sea "fruto de iniciativas voluntarias (...,] contiene elementos que resultan definitorios en el comportamiento que deben tener los actores en el Estado social de derecho" (Corte Constitucional, T-247, párrafo 7). Llama la atención el uso del verbo "deber" contrapuesto a la voluntariedad apenas invocada para describir las iniciativas de RSE. De forma similar, la Corte se ha referido a la RSE como un conjunto de programas que responden a la "necesidad" de que la empresa promueva el desarrollo de la comunidad donde opera (Corte Constitucional, T-247) o como un conjunto de medidas que no solo son deseables sino "necesarias" (Corte Constitucional, T-061). El deber y la necesidad en la implementación de políticas de RSE se explican en virtud del respaldo constitucional de esta en el ordenamiento colombiano.

Así, la Corte destaca que de los contextos en los que operan las empresas surge "una variedad de posibilidades de acción" para que la empresa pueda repercutir de manera positiva en la comunidad donde opera (Corte Constitucional, T-247, párrafo 7) y "diversas alternativas" para materializar su función social (Corte Constitucional, T-781, parágrafo 5.2.6.). La RSE no consiste entonces únicamente en ser consciente de dicha posibilidad, sino de asumirla (Corte Constitucional, T-247). Así, la realización de la función social de la empresa y la aplicación del principio de solidaridad la obligan a asumir las posibilidades existentes, entre ellas la RSE, para cumplir con los fines constitucionales. Por esto mismo, la política de RSE deberá estar alineada con dichos fines. Es claro que la RSE se concibe como una herramienta que materializa la función social de las empresas, que a la vez está alineada con los fines del Estado. Por lo tanto, la RSE concurre con el Estado en capacidades desde luego distintas. 
Sobre este punto, cabe evidenciar que, en el caso específico de los procesos de gobernanza de los recursos naturales no renovables, el llamado de la Corte es reforzado. Por un lado, la Corte ha hecho llamados específicos a las empresas que operan en el sector extractivo, especificando el espíritu en que debe enmarcarse la materialización de su función social, que "puede realizarse" mediante diversas alternativas que cumplan el contenido de los derechos humanos (Corte Constitucional, T-781). La Corte ha justificado el llamado específico a las empresas de este sector en el interés público de su actividad, del que dichas empresas derivan grandes beneficios económicos, con fuertes cargas para un grupo limitado de personas (Corte Constitucional, T-732). Por ello, a las empresas beneficiarias de concesiones del Estado les "corresponde asumir una responsabilidad social", en cuyo caso la adopción de la RSE es "no solo deseable sino necesaria" (Corte Constitucional, T-061, parágrafo 5.14.1.) y reitera: "empresas como Ecopetrol S.A. deben asumir prácticas relacionadas con el ejercicio de la responsabilidad ambiental, social y ética" (Corte Constitucional, T-061, parágrafo 5.1.4.2.). En este sentido, para el sector extractivo entre las alternativas para el cumplimiento de su constitucional función social y del principio de solidaridad, la adopción de una política de RSE es una obligación. Entre los contenidos de dicha política, también para el sector minero-extractivo, la Corte ha indicado que deben incluirse los principios constitucionales de participación ciudadana y relacionamiento permanente con las autoridades locales (Corte Constitucional, su-095).

Así, la RSE despliega lo que se ha denominado una "eficacia constitucional", en la medida en que, aunque sus fuentes de derecho internacional sean blandas, adquieren una eficacia y fuerza vinculante con base en la fuente constitucional (Correa \& Roa, 2019). Dicha fuerza vinculante se predica de los ejes de minimis, en el marco de los cuales las empresas podrán desplegar varias posibilidades de acción. Estas posibilidades implican entonces un margen de discrecionalidad para las empresas, las cuales construirán su política de RSE a partir de dicho de minimis. En este sentido, esta eficacia constitucional tiene implicaciones en sede de exigibilidad de las políticas de RSE y en sede de obligaciones de las autoridades estatales. 
En lo que respecta la exigibilidad de dichas políticas a las empresas, la eficacia constitucional de la RSE es el reflejo de la eficacia de los derechos constitucionales entre los particulares, lo cual, observa la Corte, es el fundamento de la procedibilidad de la acción de tutela contra particulares (Corte Constitucional, T-247). Esta procede en la medida en que los derechos fundamentales se consideren como vigentes, eficaces y vinculantes en las relaciones entre particulares (Corte Constitucional, T-247). Esto lo reiteró la Corte al indicar que, en la medida en que las actuaciones del particular supongan un amplio impacto social, podrá proceder la acción de tutela contra el mismo (Corte Constitucional, T-732), una reflexión realizada en el marco de la más amplia sobre deberes de la empresa en el contexto de los derechos humanos y la protección del medio ambiente. Así, podría inferirse que en la medida en que la RSE debe materializar, de manera complementaria al Estado, los fines constitucionales de este, si la empresa atenta contra los mismos o incumple su RSE, dicho actuar podría ser objeto de acción de tutela por parte del particular afectado.

En lo que respecta al rol de las autoridades del Estado frente a la RSE, en primer lugar, el juez constitucional podrá proferir órdenes a cargo de la empresa por afectación a derechos fundamentales (Corte Constitucional, T-732). En segundo, el Estado tiene un deber de prevención para evitar que las empresas vulneren derechos humanos, incluidas aquellas en las cuales participa (Corte Constitucional, T-732). Dicho deber se refleja en el llamado, también en el contexto minero-energético, a las autoridades mineras y ambientales no solo para que apoyen, sino para que "exijan rigurosamente" a las empresas o personas contratistas del sector la implementación de referentes como los principios de empresas y derechos humanos (identificados con los Principios Rectores y el Pacto Global de Naciones Unidas), que se hayan desarrollado en el país mediante el plan de acción de derechos y empresas u otras estrategias que los apliquen (Corte Constitucional, su-095).

La exigencia rigurosa que deben hacer las autoridades mineras y ambientales a las empresas en este sentido no se limita únicamente a dicha implementación, sino también a la debida diligencia social y ambiental y al respeto de los derechos humanos en cumplimiento de los postulados constitucionales (Corte Constitucional, sU-095). Es decir, 
tanto para compromisos de RSE adoptados, como para obligaciones de ley en el derecho doméstico el nivel de exigencia de las autoridades mineras y ambientales deberá ser el mismo, de manera que la obligación de vigilancia y control no diferencia entre la naturaleza ni la fuente de las normas. Aquí se presenta, nuevamente, lo que aparentemente parece ser una contradicción lógica, pues se trata de un llamado obligatorio a las autoridades del Estado a "exigir rigurosamente" lo que, por lo menos en el plano internacional, sería voluntario.

Así, la Corte refleja en su jurisprudencia la disyuntiva entre obligatoriedad y voluntariedad de las obligaciones de RSE, en el plano internacional. Coexisten pues estos dos planos de obligatoriedad y voluntariedad en cabeza de actores distintos: Estado, en el deber de vigilancia y control, y empresas, en el alcance y ejecución de la RSE, de tal manera que difícilmente podría argumentarse que en virtud de la voluntariedad de los "principios de derechos humanos y empresas" pudiera una empresa substraerse a la vigilancia y exigencia de la autoridad competente o definir arbitrariamente un contenido para la RSE.

Al contrario, lo que la Corte nos muestra, a la par con los desarrollos internacionales en el tema, es que estamos más allá de una simple "deseabilidad moral" de las conductas empresariales (Bonilla-Sanabria, 2017). En otras palabras, cobijada por el manto de una mal llamada voluntariedad, la narrativa constitucional sobre conducta empresarial responsable enfoca espacios de paradoja lógica aparente, en la que no existe arbitrariedad ni autorregulación o voluntariedad absolutas. Dichos espacios son los ejes sustanciales y procesales arriba identificados, un reflejo obligatorio de los fines constitucionales del Estado, en los que opera la RSE dirigida a dichos fines, que por tanto no puede entenderse como voluntaria en sentido absoluto.

\section{Conclusión}

El recorrido apenas realizado muestra que, si bien las fuentes de la RSE en el plano internacional se enmarcan en una categoría de voluntariedad de la que pareciera desprenderse una total libertad por parte de las empresas en su adopción, dicha apreciación, cuestionable de por sí en 
el plano internacional, debe cuestionarse aún más en el plano interno. Efectivamente, a partir del reconocimiento del carácter voluntario de la RSE, la Corte Constitucional ha desarrollado una apropiación de esta disciplina marcadamente internacional a la luz del ordenamiento jurídico colombiano. Dicha apropiación, a través de la conexidad de los fines de la RSE con los fines propios del ordenamiento constitucional en el marco del Estado social de derecho, revela dimensiones de obligatoriedad que delimitan y condicionan el alcance de la discrecionalidad propia de las empresas, las cuales, al adoptar iniciativas de RSE a la vez que manifiestan su autonomía, materializan su función social y solidaridad en el marco de los fines constitucionales del Estado. De esta manera, puede concluirse que, en Colombia, lejos de ser un ejercicio voluntario, la RSE se caracteriza por tener unos pilares de los cuales una empresa no podrá prescindir, un de minimis de obligatorio cumplimiento.

Finalmente, cabe hacer una anotación con relación a la crítica que consiste en que para que surjan contornos de obligatoriedad en la RSE, esta deberá primero ser adoptada por la empresa, lo cual podría interpretarse como un incentivo perverso para no adoptar dicha política. Ya se explicó que el sector minero-energético es una excepción a esta observación, pues la Corte ha configurado en este caso la RSE como obligatoria, en el marco de las alternativas que tienen las empresas minero-energéticas para cumplir su función social. Cabe interrogarse entonces, si el incentivo perverso opera para los otros sectores de la economía, pues en este caso la RSE es una posibilidad de acción para materializar la función social. Sin embargo, la Corte ha indicado que la RSE es no solo tomar consciencia de esta posibilidad sino asumirla y ha sido clara sobre los efectos prácticos de la función social de la actividad empresarial y la aplicación del principio de solidaridad: de estos se desprenden obligaciones y responsabilidades para las empresas, que podrían incluso ser objeto de litigio mediante acción de tutela. Adicionalmente, no deben olvidar las empresas, en particular aquellas que por una razón u otra podrían estar sometidas a litigios internacionales, que en el derecho internacional la exigibilidad de la RSE está evolucionando. De esta manera, no es plausible como política tomar la decisión de delimitar y manejar el riesgo jurídico que se deriva de la RSE omitiéndola. 
Al contrario, el manejo del riesgo jurídico que se deriva de esta implica familiarizarse con la paradoja de lo exigible en lo voluntario, definiendo claramente los límites y los fines de la política adoptada a la luz de la Constitución y siendo absolutamente conscientes de que las autoridades del Estado podrán auditar dicha política, como puede ocurrir en el sector minero-energético a la luz del llamado de la Corte. Es claro entonces que la sanción asociada a una pobre política de RSE, o a su ausencia, no es "meramente" un asunto de reputación, sino que cada vez más se aproxima a una exigibilidad de tipo legal, tanto en el plano doméstico como internacional.

\section{Referencias}

Acevedo Guerrero, J. A., Zárate Rueda, R., \& Garzón Ruíz, W. F. (2013). Estatus jurídico de la responsabilidad social empresarial (RSE) en Colombia. Revista Díkaion, 22(2), 303-332.

Aftab, Y., \& Mocle, A. (2019). Business and human rights as law: Towards justiciability of rights, involvement and remedy. Lexis Nexis.

Alfonso Monroy, M. P. (2013). Empresas y derechos humanos: una responsabilidad en construcción. Revista Facultad de Derecho y Ciencias Políticas, 43(118), 349-385.

Bernal-Fandiño, M. (2016). El principio de solidaridad como límite a la autonomía privada. Revista Jurídicas, 13(2), 60-70.

Bonilla-Sanabria, F. A. (2017). Comentarios sobre la responsabilidad social empresarial, el derecho societario y la empresa de grupo. Revista Vniversitas, (134), 21-58.

Carroll, A. B. (1999). Corporate social responsibility, evolution of a definitional construct. Business \& Society, 38(3), 268-295.

Centro Internacional de Arreglo de Diferencias Relativas a Inversiones -CIADI- (8 de diciembre de 2016). Laudo Caso CIADI ARB/07/26, Urbaser SA y Consorcio de Aguas Bilbao Bizkaia, Bilbao Biskaia ur Partzuergoa (Demandantes) y la República Argentina (Demandada). https://www.italaw.com/ sites/default/files/case-documents/italaw9627.pdf

Chinkin, C. M. (1989). The challenge of soft law: Development and change in international law. International and Comparative Law Quarterly, 38(4), 850-866. 
Colombia, Corte Constitucional. (1997, 14 de agosto). Sentencia T-375. (Eduardo Cifuentes Muñoz, MP). https://www.corteconstitucional.gov. co/relatoria/1997/t-375-97.htm

Colombia, Corte Constitucional. (2010, 15 de abril). Sentencia T-247. [Humberto Antonio Sierra Porto, MP]. https://www.corteconstitucional.gov.co/ relatoria/2010/T-247-10.htm

Colombia, Corte Constitucional. (2010, 3 de agosto). Sentencia C-608. [Humberto Antonio Sierra Porto, MP]. https://www.corteconstitucional.gov.co/ relatoria/2010/C-608-10.htm

Colombia, Corte Constitucional. (2010, 16 de noviembre). Sentencia C-915. [Humberto Antonio Sierra Porto, MP]. https://www.corteconstitucional. gov.co/relatoria/2010/C-915-10.htm

Colombia, Corte Constitucional. (2011, 3 de marzo). Sentencia T-129. [Jorge Iván Palacio Palacio, MP]. https://www.corteconstitucional.gov.co/ relatoria/2011/t-129-11.htm

Colombia, Corte Constitucional. (2014, 16 de octubre). Sentencia C-767. [Jorge Ignacio Pretelt Chaljub, MP]. https://www.corteconstitucional.gov. co/relatoria/2014/C-767-14.htm

Colombia, Corte Constitucional. (2014, 20 de octubre). Sentencia T-781. [Jorge Ignacio Pretelt Chaljub, MP]. https://www.corteconstitucional.gov. co/relatoria/2014/t-781-14.htm

Colombia, Corte Constitucional. (2016, 19 de diciembre). Sentencia T-732. [Gloria Stella Ortiz Delgado, MP]. https://www.corteconstitucional.gov. co/relatoria/2016/t-732-16.htm

Colombia, Corte Constitucional. (2017, 3 de febrero). Sentencia T-061. [Gabriel Eduardo Mendoza Martelo, MP]. https://www.corteconstitucional.gov.co/ relatoria/2017/t-061-17.htm

Colombia, Corte Constitucional. (2018, 11 de octubre). Sentencia SU-095. [Cristina Pardo Schlesinger, MP]. https://www.corteconstitucional.gov. co/relatoria/2018/SU095-18.htm

Constitución Política de Colombia. (1991). http://www.corteconstitucional. gov.co/inicio/Constitucion\%20politica\%20de\%20Colombia.pdf

Correa Henao, M., E Roa Roa J. E. (2019). Derechos humanos, responsabilidad social empresarial y principios Ruggie en la jurisprudencia constitucional colombiana. En T. Martínez J. (Ed.), Derechos humanos y la actividad empresarial en Colombia: implicaciones para el Estado social de derecho (pp. 152-194). Universidad Externado de Colombia. 
Fischer-Lescano A., E Teubner, G. (2004). Regime collisions: The vain search for legal unity in the fragmentation of global law. Michigan Journal of International Law, 25(1), 999-1046.

Freeman, R. E., Harrison, J. S., Wicks, A. C., Parmar, B., E de Colle, S. (2010). Stakeholder theory. The state of the art. Cambridge University Press.

Friedman, M. (13 de septiembre de 1970). The social responsibility of business is to increase its profits. The New York Times Magazine. http://umich. edu/ thecore/doc/Friedman.pdf

Gatti, L., Seele P., E Rademacher L. (2019). Grey zone in - greenwash out. A review of greenwashing research and implications for the voluntarymandatory transition of CSR. International Journal of Corporate Social Responsibility 4 (6), 1-15. https://doi.org/10.1186/s40991-019-0044-9

Klabbers, J. (1996). The redundancy of soft law. Nordic Journal of International law, 65(2), 167-182. https://doi.org/10.1163/15718109620294889.

Latapí Agudelo, M. A., Jóhannsdóttir, L., E Davídsdóttir, B. (2019). A literature review of the history and evolution of corporate social responsibility. International Journal of Corporate Social Responsibility 4(1), 1-23. https://doi. org/10.1186/s40991-018-0039-y

Linderfalk, U. (2015). Cross-fertilisation in international law. Nordic Journal of International Law 84(3), 428-455. https://doi.org/10.1163/1571810708403004

López, C. (2019). El proyecto revisado de un tratado sobre empresas y derechos humanos: mejoras innovadoras y perspectivas más claras. Investment Treaty News, 10(4), 12-15.

Mitchell, R. K., E Lee J. H. (2019). Stakeholder identification and its importance in the value creating system of stakeholder work. En J. S. Harrison, J. B. Barney, R. E. Freeman, E R. A Philips (Ed.), The Cambridge handbook of stakeholder theory (pp. 54-73). Cambridge University Press.

Morgenstein-Sánchez, W. I. (2019). La responsabilidad social de la empresa y el derecho de la competencia en Colombia: Una visión desde la economía social de mercado y la multi-stakeholder theory. Revista Republicana, 27, 69-87.

Muchlinski, P. T. (2011). International corporate social responsibility and international law. En T. Weiler E F. Baetens, (Ed.), New directions in international economic law: In memoriam Thomas Wälde (pp. 223-244). Martinus Nijhoff Publishers.

Naciones Unidas. (2000). Pacto global. https://www.unglobalcompact.org/

Naciones Unidas. (2003). Normas sobre las responsabilidades de las empresas transnacionales y otras empresas comerciales en la esfera de los derechos humanos. 
https://observatoriorsc.org/normas-sobre-las-responsabilidades-de-lasempresas-transnacionales-y-otras-empresas-comerciales-en-la-esfera-delos-derechos-humanos/

Naciones Unidas (2011). Informe del Representante Especial del Secretario General para la cuestión de los derechos humanos y las empresas transnacionales y otras empresas, John Ruggie. Principios Rectores sobre las empresas y los derechos humanos: puesta en práctica del marco de las Naciones Unidas para "proteger, respetar y remediar" (A/HRC/17/31). https://documents-dds-ny.un.org/doc/ UNDOC/GEN/G11/121/93/PDF/G1112193.pdf?OpenElement

Naciones Unidas. (2014). Elaboration of an international legally binding instrument on transnational corporations and other business enterprises with respect to human rights (A/HRC/RES/26/9). https://ap.ohchr.org/documents/dpage_e. aspx?si=A/HRC/RES/26/9

Neuwirth, R. J. (2017). Global law and sustainable development: change and the "developing-developed country" terminology. The European Journal of Development Research, 29 (5), 911-925.

OCDE. Guidelines for international enterprises. http://www.oecd.org/daf/inv/ mne/48004323.pdf

Okoye, A. (2016). Legal approaches and corporate social responsibility. Towards a llewelyn's law-jobs approach. Routledge.

Picciotto, S. (2002). Introduction: Reconceptualizing regulation in the era of globalization. Journal of Law and Society, 29(2), 1-11.

Quinche Ramírez, M. F. (2009). Derecho constitucional colombiano de la Carta de 1991 y sus reformas. Editorial Universidad del Rosario.

Rodríguez Garavito, C. (2017). Business and human rights: Beyond the end of the beginning. En C. Rodríguez Garavito (Ed.), Business and human rights: Beyond the end of the beginning (pp. 11-45). Cambridge University Press.

Ruggie J. G., (2017). Hierarchy or ecosystem? Regulating the human rights risks of multinational enterprises. En C. Rodríguez Garavito (Ed.), Business and human rights (pp. 46-61). Cambridge University Press.

Tamvada M. (2020). Corporate social responsibility and accountability: a new theoretical foundation for regulating CSR. International Journal of Corporate Social Responsibility, 5(2), 1-14. https://doi.org/10.1186/s40991-019-0045-8

Teubner, G. (2017). Corporate codes in the varieties of capitalism: How their enforcement depends on the differences among production regimes. Indiana Journal of Global Legal Studies, 24(1), 81-97.

Tomuschat, C. (2014). Human rights, between idealism and realism. Oxford University Press. 